\title{
A Stochastic-Process-Based Method for Assessing Frequency Regulation Ability of Power Systems with Wind Power Fluctuations
}

\author{
Y. Guo ${ }^{1, *}$, Q. Wang ${ }^{1}$, D. Zhang ${ }^{1}$, D. Yu ${ }^{2}$, and J. Yu ${ }^{1}$ \\ ${ }^{1}$ School of Electrical Engineering and automation, Harbin Institute of Technology, Harbin, 150001 China \\ ${ }^{2}$ Korea School of Energy Science and Engineering, Harbin Institute of Technology, Harbin, 150001 China
}

Received March 30, 2016; revised January 18, 2018; accepted March 29, 2018; published online September 23, 2018

\begin{abstract}
The increasing penetration of wind power raises the problem of maintaining grid frequency stability. The purpose of this paper is to quantitatively describe the relationship between wind power fluctuations and grid frequency deviation. The fluctuation characteristics of wind power are analyzed in multi-time scales by wavelet methods. Then, a mathematical model representing wind power fluctuations is established. Using this model and the frequency response transfer function of power system, the frequency deviation can be obtained. A coefficient to measure the frequency regulation ability (FRA) of power system is defined as the ratio of wind power fluctuations to frequency deviations. Based on a series of calculation results of FRA in a two-area power system with large-scale wind power integration, a strategy for deploying appropriate thermal units participating in frequency regulation is proposed. In this strategy, the future frequency deviation of the system can be assessed, which helps operators to adjust the deployment of thermal units reasonably. Case studies show that this strategy can be widely applied in decreasing grid frequency deviation caused by wind power fluctuations.
\end{abstract}

Keywords: frequency deviation, frequency regulation ability, frequency regulation, wind power fluctuation, wavelet methods, units deployment

\section{Introduction}

As a renewable and environmental friendly energy source, wind generation has recently witnessed accelerated expansion throughout the world (Yao, et al., 2012; Wu et al., 2016; Ji, et al., 2016). However, due to the stochastic nature of wind, the resulting fluctuations in intermittent wind energy substantially handicap large-scale integration of wind power into regional power grids (Tan et al., 2013; Suo et al., 2013; Hu et al., 2014). In order to maintain stable operations, electric power utilities need some technical support to suppress the fluctuations of wind power (Bouffard and Galiana, 2008; Luickx et al., 2009; Al-Awami and El-Sharkawi, 2011).

Wind power fluctuations would cause frequency deviation from the standard. Excessive grid frequency deviation could affect normal system operations and, may even cause power system splitting. In this case, it is important to find the quantitative relationship between wind power fluctuations and grid frequency deviation. By analyzing this relationship, strategies for wind power smoothing can be made and the risk of excessive frequency deviation can be avoided.

To figure out the relationship, the primary concern is the modeling of wind power fluctuation characteristic. Many ap-

\footnotetext{
* Corresponding author. Tel.: +86 45186413641; fax: +86 45188192192 .
}

E-mail address: guoyufenghit@163.com (Y. Guo).

ISSN: 1726-2135 print/1684-8799 online

(C) 2018 ISEIS All rights reserved. doi: 10.3808/jei.201800394 proaches have been proposed to quantify wind power fluctuations in published studies, such as the probabilistic forecasting method (Boutsika and Santoso, 2012; Khosravi et al., 2013; Can et al., 2014), and the frequency and time domain analysis method (Sorensen et al., 2007; Nazir and Bouffard, 2012). In this paper, the characteristics of wind power fluctuations are analyzed by the wavelet methods in multi-time scales. Figure 1 describes three wavelet components of wind power. Figure 1a presents the low frequency component of wind power. Figure $1 \mathrm{~b}$ and Figure 1c present minute time-scale and second time-scale fluctuating component respectively. As shown in Figure 1, there is a significant relationship between low frequency component and fluctuating components (Kisi et al., 2013). Based on this relationship, a wind power fluctuation model is constructed.

In terms of assessing grid frequency deviation, the methodology used in (C. Luo, 2006; C. Luo, 2007; H. Banakar, 2008) consisted of modeling a test power system in a simulation software. The wind farm output power fluctuation is represented by a sinusoidal time varying signal. Conclusion regarding robustness against wind power fluctuation in (Luo and Ooi, 2006; Luo et al., 2007; Banakar et al., 2008) is formed until wind penetration reaches $27.6 \%$. Such conclusions are drawn only based on the analysis of deterministic wind power fluctuations. However, the stochastic power fluctuation of the wind farm is not included (Luo and Ooi, 2006; Luo et al., 2007; Banakar et al., 2008). In Lin et al. (2012), a method based on "Time-Frequency Transformation" is presented, where the stochastic wind power fluctuation is taken into full account. In this 
paper, a stochastic analytical approach is applied for analyzing the relationship between the stochastic wind power and grid frequency deviation. Then, a new coefficient to measure the frequency regulation ability of power system is defined.
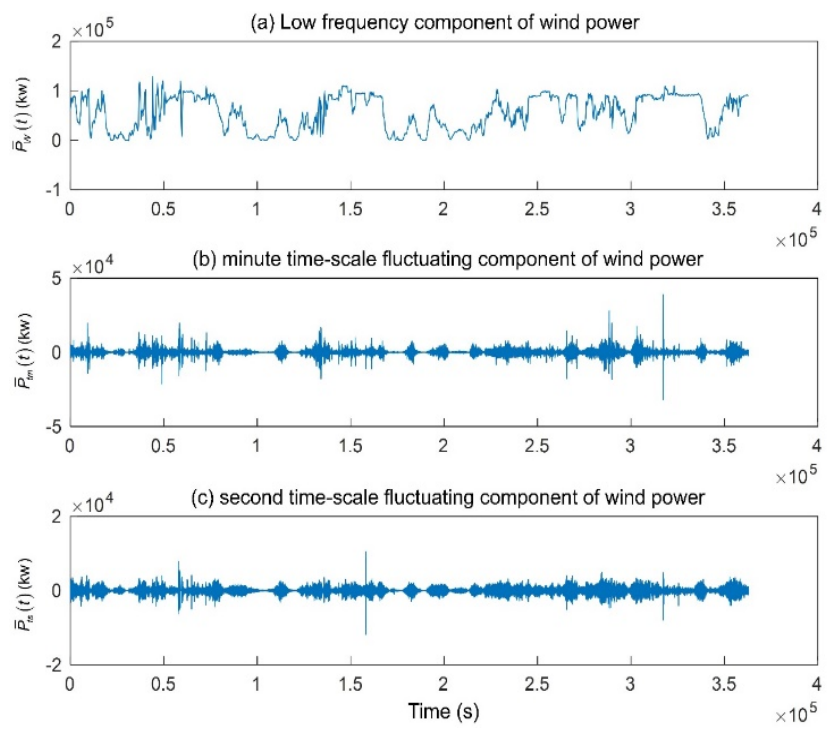

Figure 1. Modulation effect between 15-minute moving average wind power $\bar{P}_{w}(t)$ and fluctuations component $\sigma_{m}(t)$, $\sigma_{s}(t)$. (a) low frequency component of wind power; (b) minute time-scale fluctuating component of wind power; (c) second time-scale fluctuating component of wind power.

To reduce the risk of excessive frequency deviation, reasonable power system operating strategies should be formulated. Many studies have shown that wind power fluctuations can be smoothed by appropriate control and deployment of conventional thermal units. The effect of fluctuating wind power on frequency deviation is studied in (Sorensen et al., 2007) and operation reserve is suggested to increase with the increase of wind power scale. In (Ortega-Vazquez and Kirschen, 2009; Xia et al., 2013), methods about how to determine and deploy the system operation reserve to compensate the fluctuation of wind power are presented. In addition, system operators should dictate the dynamic requirements of the scheduled reserve capacity for conducting the primary and secondary frequency regulation tasks at the system level (Galiana et al., 2005; Bouffard and Ortega-Vazquez, 2011). In general, most thermal units are participating in primary frequency regulation and only a few AGC units would participate in secondary frequency regulation. In order to avoid excessive grid frequency deviation, both system operation reserve and the deployment of different kinds of thermal units should be taken into consideration. In this paper, a FRA based strategy for appropriately deploying thermal units participating in frequency regulation is proposed. Case studies are conducted to show how this strategy is implemented to reduce excessive frequency deviation caused by wind power fluctuations.
The main contributions of this paper are:

1) a novel multi-timescales wind power fluctuation model based on wavelet multi-resolution signal decomposition and reconstruction method is proposed;

2) a method to analyze the relationship between the stochastic wind power and grid frequency deviation using the stochastic analytical approach is proposed. A new coefficient to measure the FRA of power system is defined;

3) a strategy for thermal units deployment to avoid excessive frequency deviation caused by wind power fluctuations is conducted.

The remainder of the paper is organized as follows. In Section 2, a time domain model of wind power fluctuation is presented. The theoretical foundation and procedure for calculateing FRA are shown in detail in Section 3. FRA under different conditions is calculated and a strategy for deploying thermal units participating in frequency regulation is presented in Section 4. Case studies are conducted in Section 5. Conclusions are finally drawn in Section 6.

\section{Modeling of the Wind Power Fluctuations}

\subsection{Modulation Effect of Wind Power Low Frequency Component}

In this paper, the characteristic of wind power fluctuations mainly refers to the fluctuation magnitude in different time scales. The analysis is based on the actual wind power data sampled every 5-second. Measured wind power time series $P_{w}(t)$ are decomposed into three parts by 8-level discrete wavelet decomposition and reconstruction methods (Rahmani, 2015). The three parts of $P_{w}(t)$ are low frequency component $\bar{P}_{w}(t)$, minute time-scale wind power fluctuation component $P_{t m}(t)$ and second time-scale wind power fluctuation component $P_{t s}(t)$.

As shown in Figure 1, there is a significant relationship between the low frequency component $\bar{P}_{w}(t)$ and the fluctuation components $P_{t}(t)=P_{t m}(t)+P_{t s}(t)$. The amplitude of $P_{t m}(t)$ and $P_{t s}(t)$ are large when the amplitude of $\bar{P}_{w}(t)$ is large. When the amplitude of $\bar{P}_{w}(t)$ is nearly zero, the amplitudes of $P_{t m}(t)$ and $P_{t s}(t)$ are also very small.

To analyze the relationship between each component in more detail, standard deviations of $P_{t}(t), P_{t m}(t)$ and $P_{t s}(t)$ are marked as $\sigma(t), \sigma_{m}(t)$ and $\sigma_{s}(t)$. Then, $\bar{P}_{w}(t)$ and its corresponding $\sigma(t), \sigma_{m}(t)$ and $\sigma_{s}(t)$ are sorted by amplitude. The curve fitting result of $\bar{P}_{w}(t)$ and $\sigma(t)$ is shown in Figure 2.

As shown in Figure 2, the relation between $\sigma(t)$ and $\bar{P}_{w}(t)$ can be described by a power exponential function. It can be inferred that the amplitude of $P_{t}(t)$ is modulated by $\bar{P}_{w}(t)$.

The functional relation between $\sigma(t)$ and $\bar{P}_{w}(t)$ can be obtained by fitting the empirical data:

$\sigma=a \cdot \bar{P}_{W}^{b}+c$ 


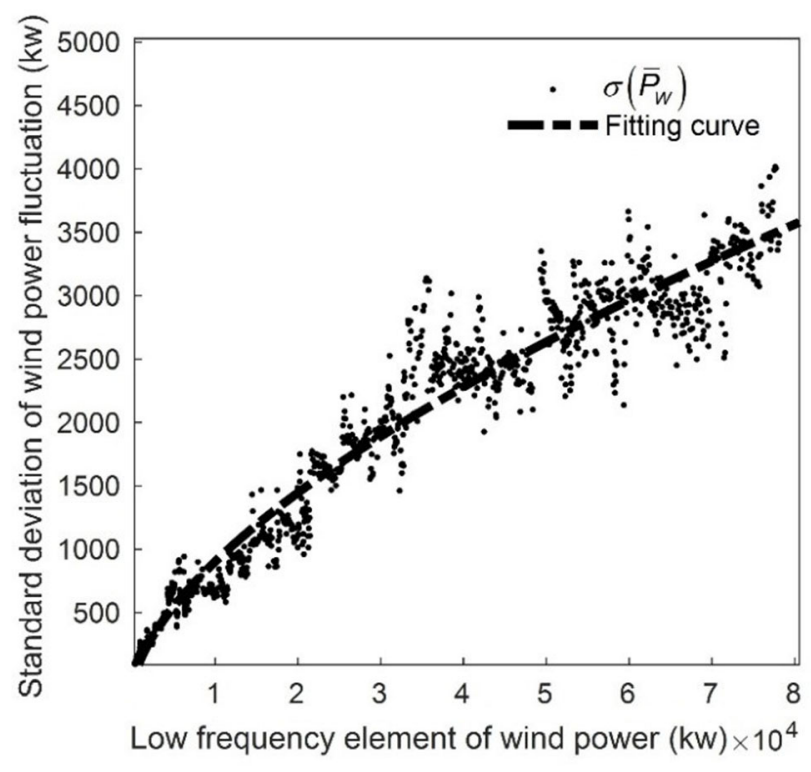

Figure 2. Fitting curve between $\bar{P}_{w}(t)$ and $\sigma(t)$.

\subsection{Minute-scale Wind Power Fluctuation Model}

The relative variation between $\sigma_{m}(t)$ and $\bar{P}_{w}(t)$, which is defined as $\sigma_{r m}(t)$, can be calculated by the ratio of $\sigma_{m}(t)$ to $\bar{P}_{w}(t)$. The correspondence between $\sigma_{r m}(t)$ and $\bar{P}_{w}(t)$ in per unit value is shown in Figure 3.

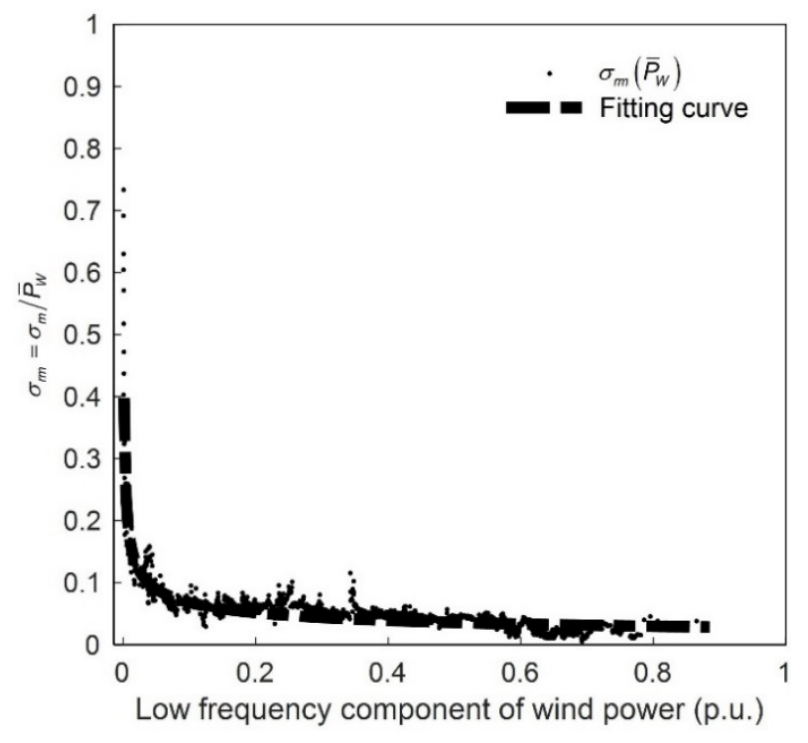

Figure 3. Fitting curve between $\sigma_{m}(t)$ and $\bar{P}_{w}(t)$.

From Figures 2 and 3, $\sigma_{m}(t)$ increases, while $\sigma_{r m}(t)$ decreases as $\bar{P}_{w}(t)$ increases. In addition, as $\bar{P}_{w}(t)$ increases, $\sigma_{r m}(t)$ decreases in a gradually gentler way. By curve fitting, this relation can be expressed as an approximated power function:

$$
\sigma_{r m}=\sigma_{m} / \bar{P}_{W}=a_{m} \cdot \bar{P}_{W}^{b_{m}}+c_{m}
$$

where $a_{m}, b_{m}$, and $c_{m}$ are fitting coefficients.

In order to compare the fluctuation characteristics of wind power from different wind farms, the wind power data from northeast and northwest of China have been analyzed. The curve fitting results are shown in Table 1.

Table 1. Curve Fitting Results of Different Wind Farms

\begin{tabular}{cl}
\hline $\begin{array}{c}\text { Sequence number } \\
\text { of wind farm }\end{array}$ & Curve fitting results \\
\hline 1 & $\sigma_{r m}=0.0086 \bar{P}_{W}^{-0.5927}+0.0076$ \\
2 & $\sigma_{r m}=0.0123 \bar{P}_{W}^{-0.7526}+0.0043$ \\
3 & $\sigma_{r m}=0.0115 \bar{P}_{W}^{-0.4665}+0.0129$ \\
4 & $\sigma_{r m}=0.0201 \bar{P}_{W}^{-0.6752}+0.0274$ \\
5 & $\sigma_{r m}=0.0251 \bar{P}_{W}^{-0.5307}+0.0108$ \\
\hline
\end{tabular}

As shown in Table 1, the values of $a_{m}, b_{m}$, and $c_{m}$ vary from different wind farm locations and topographies. The maximum values of $a_{m}$ and $c_{m}$ are 3 and 6 times larger than their minimum values respectively. The variations of $b_{m}$ have significant influence on $\sigma_{r m}$ only when $\bar{P}_{w}$ is small.

From Equation (2), the standard deviation of minute timescale wind power fluctuations can be forecasted. In power systems, secondary frequency regulation is mainly responsible for frequency deviation in minute time-scale. Thus, this model is suitable for analyzing the influence of wind power fluctuation on secondary frequency regulation.

\subsection{Second-scale Wind Power Fluctuation Model}

The relationship between $\sigma_{r s}(t)$ and $\bar{P}_{w}(t)$ can be seen from Figure 4 using above method.

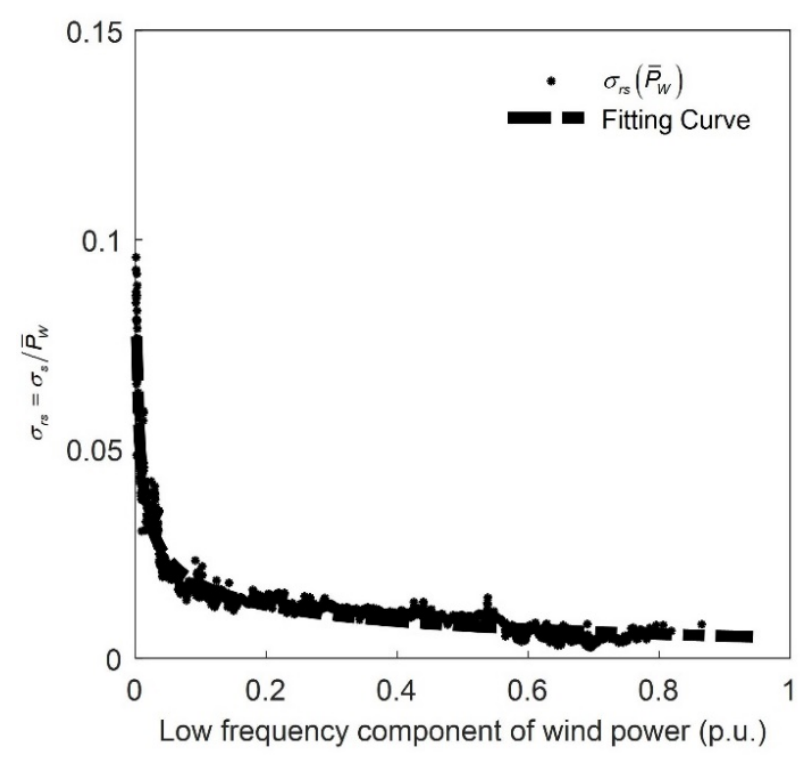

Figure 4. Fitting curve between $\sigma_{r s}(t)$ and $\bar{P}_{w}(t)$.

The relationship between $\sigma_{r s}(t)$ and $\bar{P}_{w}(t)$ can be expressed as an approximate power function: 
$\sigma_{r s}=\sigma_{s} / \bar{P}_{W}=a_{s} \cdot \bar{P}_{W}^{b_{s}}+c_{s}$

where $a_{s}, b_{s}$, and $c_{s}$ are fitting coefficients.

The curve fitting results are shown in Table 2 using the same data from five wind farms.

Table 2. Curve Fitting Results of Different Wind Farms

\begin{tabular}{cc}
\hline $\begin{array}{c}\text { Sequence number of } \\
\text { wind farm }\end{array}$ & Curve fitting results \\
\hline 1 & $\sigma_{r s}=0.0042 \bar{P}_{W}^{-0.5409}+0.0022$ \\
2 & $\sigma_{r s}=0.0059 \bar{P}_{W}^{-0.3462}+0.0037$ \\
3 & $\sigma_{r s}=0.0054 \bar{P}_{W}^{-0.4188}+0.0085$ \\
4 & $\sigma_{r s}=0.0069 \bar{P}_{W}^{-0.7408}+0.0234$ \\
5 & $\sigma_{r s}=0.0102 \bar{P}_{W}^{-0.3612}+0.0062$ \\
\hline
\end{tabular}

Results in Figures 3 and 4 show that the method above is useful in modeling wind power fluctuations. The approximate power function universally applicable to describe wind power fluctuations from different wind farms in various time-scales. The coefficients in the functions can be obtained by processing the historical data from different wind farms.

\section{Method for Assessing System Frequency Regulation Ability Consider Wind Power Fluctuations}

After analyzing the fluctuation characteristic of wind power, this section outlines the method for assessing frequency regulation ability of power systems with wind power fluctuations.

\subsection{Basic Mathematical Theories for Stochastic Process Analysis}

In stochastic system analysis, the stochastic time series input signal $x(t)$ is divided into segments $x_{k}(t)(k=1,2, \ldots, n)$, with equal time duration. Each segment undergoes Fast Fourier transform (FFT) to form frequency domain inputs $x_{k}(f)$. In a stochastic system, the relation $Y(f)=H(f) \cdot X(f)$ cannot be used. Instead, a similar relation exists based on the concept of power spectrum density (PSD) theory. The PSD $S_{x}(f)$ is determined as (Sorensen et al., 2007):

$$
S_{x}(f) \Delta f=E\left(X(f) \cdot X^{*}(f)\right)
$$

where $\Delta f$ is the sample frequency, $X(f)$ represents a vector consisting of $X_{k}(f)$, the * operator denotes the complex conjugate of $X(f)$ and $E(\cdot)$ represents ensemble average. $E(\cdot)$ is calculated as the average of the product of $X_{k}(f)$ and $X_{k}^{*}(f)$ in frequency domain as:

$$
E\left(X_{k}(f) \cdot X^{*}(f)\right)=\frac{1}{n} \sum_{i=1}^{n} X_{i}(f) \cdot X_{i}^{*}(f)
$$

For an input PSD, $S_{x}(f)$, the output PSD, $S_{y}(f)$ is obtainable from the following formula:

$$
S_{y}(f)=|H(f)|^{2} S_{x}(f)
$$

The input signal $x(t)$ is assumed to be a zero-mean random stationary process. As a stationary process, the variance of output $y(t)$ is obtainable from Equation (7).

$\sigma_{y}^{2}=\frac{1}{2 \pi} \int_{-\infty}^{\infty} S_{y}(f) d f$

\subsection{Definition of Primary Frequency Regulation Ability}

Primary frequency regulation in a power system is a dynamic stochastic process that can automatically respond to frequency deviation within second time-scale (Daren and Yufeng, 2004). The variance of a stochastic variable is an illustration of the amplitude of variation. Then, a coefficient called primary frequency regulation ability (PFRA) is introduced as follows:

$$
\begin{aligned}
& D_{\text {PFRA }}= \\
& \sqrt{\frac{\text { variance of wind power fluctuation (seconds) }}{\text { variance of grid frequency deviation }}}
\end{aligned}
$$

This coefficient represents the influence of second timescale wind power fluctuations on system frequency deviation.

The frequency response $|H(f)|$ of the power system is constructed by transfer functions of many components. $D_{P F R A}$ is affected by transfer function of each component. Since the goal of frequency regulation is to maintain system frequency deviation within a certain range $\pm f_{T}$, Equation (8) can be transformed into Equation (9):

$$
\begin{aligned}
& \sigma_{f 1}= \\
& \frac{\sqrt{\text { variance of wind power fluctuation (seconds) }}}{D_{P F R A}}
\end{aligned}
$$

where $\sigma_{f 1}(t)$ is the frequency deviation caused by second timescale wind power fluctuation. $\sigma_{f T}\left(\sigma_{f T}=3 \cdot f_{T}\right)$ is the threshold val-ue of frequency deviation.

\subsection{Definition of Secondary Frequency Regulation Ability}

Secondary frequency regulation in a power system mainly deals with minute time-scale wind power fluctuations. Similarly, a coefficient named secondary frequency regulation ability (SFRA) is defined as follows:

$$
\begin{aligned}
& D_{\text {SFRA }}= \\
& \sqrt{\frac{\text { variance of wind power fluctuation (minute) }}{\text { variance of grid frequency deviation }}}
\end{aligned}
$$


$D_{S F R A}$ represents the ratio of variance of minute time-scale wind power fluctuation and variance of frequency deviation during a period of time only with secondary frequency regulation effect. Equation (10) can be transformed into Equation (11) with the above method:

$$
\begin{aligned}
& \sigma_{f 2}= \\
& \frac{\sqrt{\text { variance of wind power fluctuation (minute) }}}{D_{S F R A}}
\end{aligned}
$$

where $\sigma_{f 2}$ is the frequency deviation caused by minute timescale wind power fluctuation.

Based on the above analysis, a method for assessing frequency regulation ability of power systems with wind power fluctuations is illustrated in Figure 5.

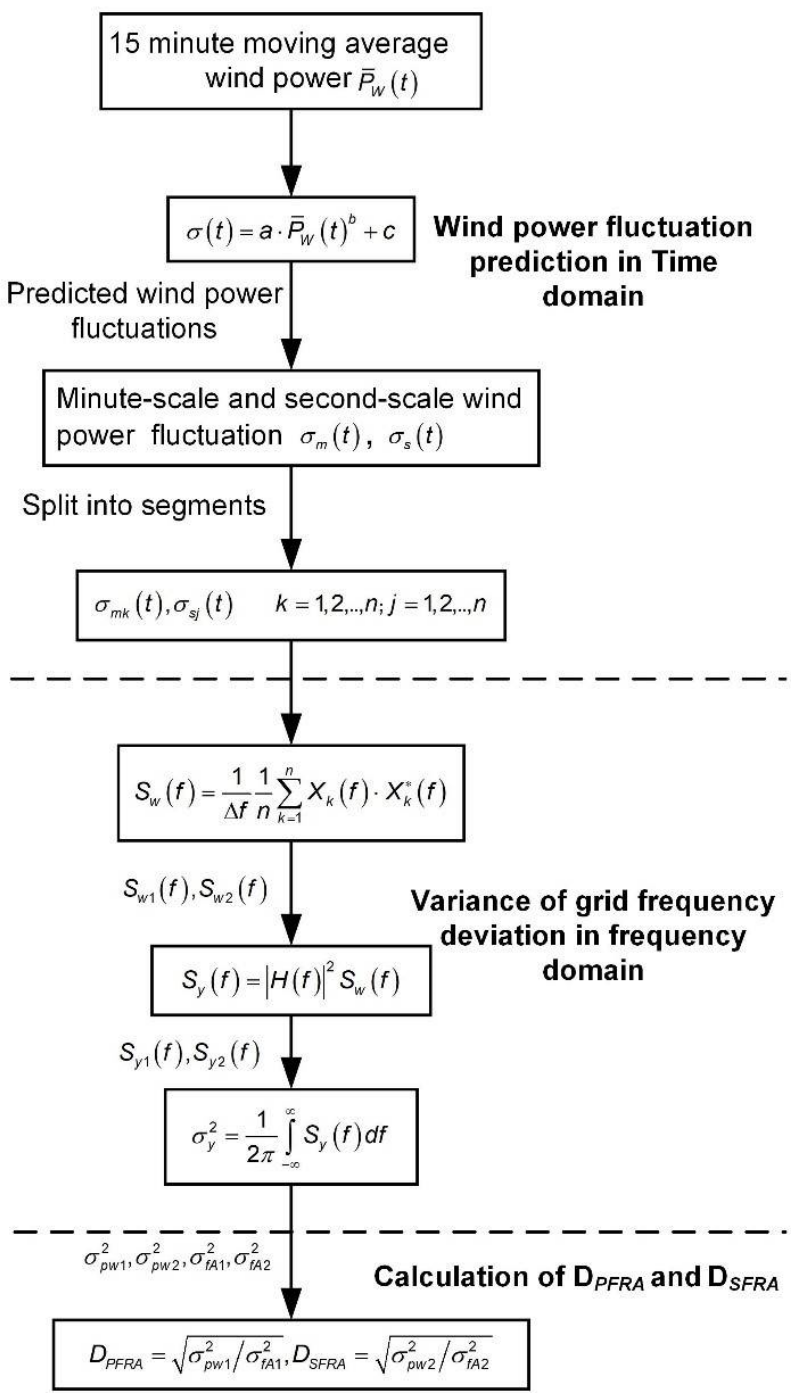

Figure 5. Overview of the calculation process of $D_{P F R A}$ and DSFRA.
Three major steps of the method are outlined as follows: (1) The minute and second time-scale wind power fluctuation time series $x_{m}(t)=\sigma_{m}(t)$ and $x_{s}(t)=\sigma_{s}(t)$ are predicted from Equations (2) and (3). Then, $X_{m k}(f)$ and $X_{s k}(f)$ are obtained via FFT. (2) By substituting $X_{m k}(f)$ and $X_{s k}(f)$ into Equations (4) (7) respectively, the variance of grid frequency deviation caused by minute and second time-scale wind power fluctuation are obtained. (3) Finally, the DPFRA and DSFRA are calculated to quantify frequency regulation ability of power systems with wind power fluctuations in different time-scales.

\section{System Frequency Regulation Ability Calculation in a Case Study}

A two-area power system for case study is presented in Figure 6 (Elgerd and Fosha, 1970).

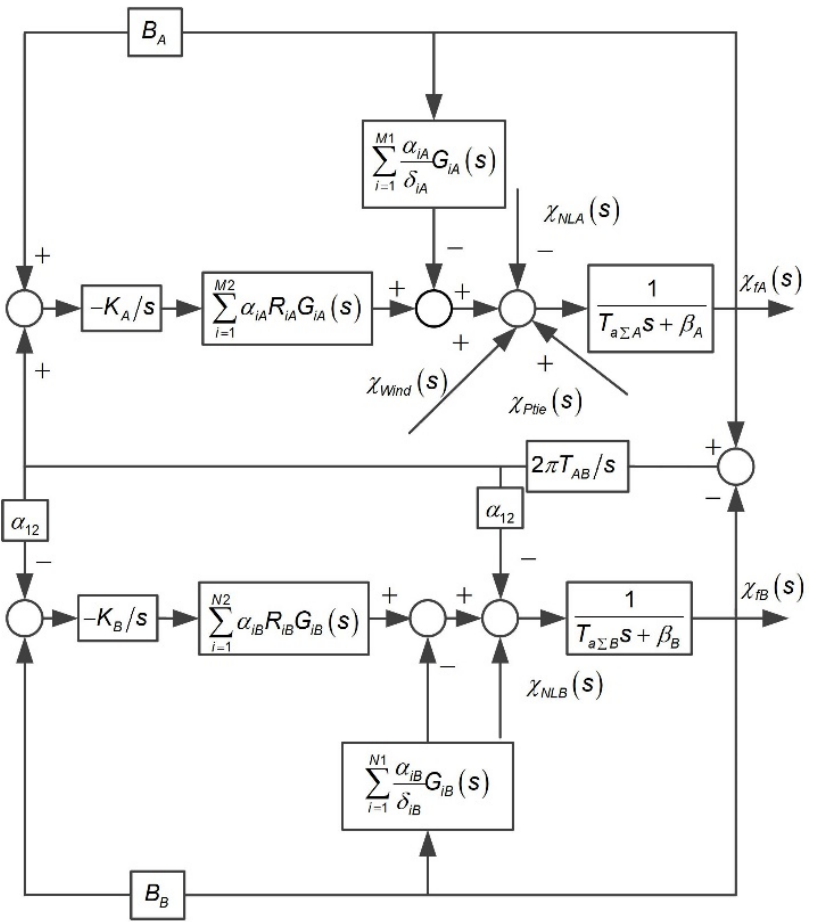

Figure 6. Block diagram of two-area system with AGC control include wind power.

This paper focuses on the influence of wind power fluctuations on system frequency. Thus, the load stochastic fluctuation and faults of power system are not considered. Area $A$ comprises of a wind farm and thermal plants, whereas area $B$ comprises only of thermal plants. The two areas are connected by a tie-line. Data used for analysis is a one-month record of the actual wind power data sampled every 5 seconds from a wind farm located in China. This section is organized as follows:

1) Analytical expressions of DPFRA and DSFRA in a two-area power system are given and calculated.

2) The influence of different factors on frequency regulation ability of power systems are given and calculated. 


\subsection{Derivation of $D_{P F R A}$ and $D_{S F R A}$}

Step I: The first step is to obtain the variance of stochastic wind power fluctuation.

The 15-minute moving average wind power time series $\bar{P}_{w}(t)$ is taken as the input of Equations (2) and (3). The minute and second time-scale wind power fluctuation components are predicted from Equations (2) and (3). In this case, the coefficient values are $a_{m}=0.0115, b_{m}=-0.4665, c_{m}=0.0129, a_{s}=$ $0.0054, b_{s}=-0.4188, c_{s}=0.0085$.

The minute time-scale fluctuation component $\sigma_{m}(t)$ is split into 5-minute segments and second time-scale fluctuation component $\sigma_{s}(t)$ is divided into 30 -second segments. Each segment undergoes FFT to yield $X(f)$ in frequency domain. The period of each segment is $T_{\text {seg. }}$. Thus, the corresponding frequency $\Delta f$

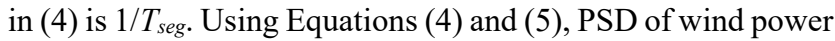
fluctuations $S_{w}(f)$ are computed.

By substituting $S_{w}(f)$ into Equations (7), the variance of wind power fluctuations can be obtained by:

$\sigma_{p w}^{2}=\frac{1}{2 \pi} \int_{-\infty}^{\infty} S_{w}(f) d f$

Step II: This step is to obtain the variance of frequency deviation in area $A$ caused by wind power fluctuations and the frequency response transfer function $|H(f)|$.

Since the focus is the impact of wind power fluctuations on system frequency deviation. The load fluctuation is assumed to be zero in Figure 6.

1) Primary frequency regulation: In this subsection, only the primary frequency regulation effect is taken into consideration. The frequency deviation $\chi_{f A 1}(s)$ in area $A$ caused by second time-scale wind power fluctuation $\chi_{p w 1}(s)$ can be expressed as:

$\chi_{f A 1}(s)=-\frac{1}{T_{a \sum A} s+\beta_{A}+G_{A}(s)} \chi_{p w 1}(s)$

and $G_{A}(s)=\sum_{i=1}^{M 1} \frac{\alpha_{i A}}{\delta_{i A}} G_{i A}(s)$.

The primary frequency response transfer function $H_{p}(s)=$ $\chi_{f A 1}(s) / \chi_{p w 1}(s)$ has been obtained through Equation (13).

$H_{p}(s)=\frac{1}{T_{a \sum A} s+\beta_{A}+G_{A}(s)}$

Where $S_{w 1}(f)$ is the PSD of second time-scale wind power fluctuation. By substituting $S_{w 1}(f)$ and Equation (14) into Equation (6), $S_{y 1}(f)$, the PSD of grid frequency deviation caused by second time-scale wind power fluctuation is predicted.
$S_{y 1}(f)=\left|\frac{1}{T_{a \sum A} s+\beta_{A}+G_{A}(s)}\right|^{2} \cdot S_{w 1}(f)$

where $\chi_{f A 1}$ is a deviation variable with a mean value of 0 . By substituting Equation (15) into Equation (7), the variance of $\chi_{f A 1}(s)$ is computed as:

$$
\begin{aligned}
\sigma_{f A 1}^{2} & =\frac{1}{2 \pi} \int_{-\infty}^{\infty}\left|\chi_{f A 1}(f)\right|^{2} d f \\
& =\frac{1}{2 \pi} \int_{-\infty}^{\infty}\left|\frac{1}{T_{a \sum A} s+\beta_{A}+G_{A}(s)}\right|^{2} S_{w 1}(f) d f
\end{aligned}
$$

2) Secondary frequency regulation: In this subsection, only the secondary frequency regulation effect is taken into consideration. The frequency deviation in area $A$ caused by minute time-scale wind power fluctuation $\chi_{p w 2}(s)$ can be expressed in the same form as Equation (13), where $G_{A}(s)$ is changed.

The secondary frequency response transfer function $H_{s}(s)$ $=\chi_{f A 2}(s) / \chi_{p w 2}(s)$ can be expressed as:

$H_{s}(s)=\frac{1}{T_{a \sum A} s+\beta_{A}+B_{A} / s \cdot e^{-s \tau_{d}} \cdot G_{A}(s)}$

Where $S_{w 2}(f)$ is the PSD of minute time-scale wind power fluctuation. By substituting $S_{w 2}(f)$ and Equation (17) into Equation (6), $S_{y 2}(f)$, the PSD of grid frequency deviation caused by minute time-scale wind power fluctuation can be predicted:

$$
\begin{aligned}
& S_{y 2}(f)= \\
& \left|\frac{1}{T_{a \sum A} s+\beta_{A}+B_{A} / S \cdot e^{-s \tau_{d}} \cdot G_{A}(s)}\right|^{2} \cdot S_{w 2}(f)
\end{aligned}
$$

The variance of $\chi_{f A 2}(s)$ is computed based on Equation (7):

$$
\begin{aligned}
\sigma_{f A 2}^{2} & =\frac{1}{2 \pi} \int_{-\infty}^{\infty}\left|\chi_{f A 2}(f)\right|^{2} d f \\
& =\frac{1}{2 \pi} \int_{-\infty}^{\infty}\left|\frac{1}{T_{a \Sigma A} s+\beta_{A}+B_{A} / s \cdot e^{-s \tau_{d}} \cdot G_{A}(s)}\right|^{2} S_{w 2}(f) d f
\end{aligned}
$$

Step III: The last step is to obtain the analytical expression of $D_{P F R A}$ and $D_{S F R A}$ defined in Equations (8) and (10). 
By substituting Equations (12) and (16) into Equation (8), the primary frequency regulation ability can be expressed as:

$$
\begin{aligned}
D_{P F R A} & =\sqrt{\sigma_{p w 1}^{2} / \sigma_{f A 1}^{2}} \\
& =\sqrt{\frac{\int_{-\infty}^{\infty} S_{w 1}(f) d f}{\left.\int_{-\infty}^{\infty} \mid-1 /\left[T_{a \Sigma A} j f+\beta_{A}+G_{A}(j f)\right]\right]^{2} \cdot S_{w 1}(f) d f}}
\end{aligned}
$$

where $\alpha_{p f r}$ and $\delta_{i A}$ are the two main factors which influent the value of $D_{P F R A}$.

By substituting Equations (12) and (19) into Equation (10), the secondary frequency regulation ability can be expressed as:

$$
\begin{aligned}
D_{\text {SFRA }} & =\sqrt{\sigma_{p w 2}^{2} / \sigma_{f A 2}^{2}} \\
& =\sqrt{\frac{\int_{-\infty}^{\infty} S_{w 2}(f) d f}{\int_{-\infty}^{\infty}\left|-\frac{1}{T_{a \Sigma A} j f+\beta_{A}+B_{A} \cdot\left(K_{A} / j f\right) \cdot G_{A}(j f)}\right|^{2} \cdot S_{w 2}(f) d f}}
\end{aligned}
$$

where $G_{A}(s)=\sum_{i=1}^{M 2} \alpha_{i A} R_{i A} G_{i A}(s), S_{w 2}(f)$ is the PSD of minute time-scale wind power fluctuation; $\alpha_{s f r}$ and $K_{A}$ are the two main factors which influent the value of $D_{S F R A}$.

\subsection{Calculation of $D_{P F R A}$ and $D_{S F R A}$ of a Two-area Power System with Wind Power Fluctuations}

In this section, $D_{P F R A}$ and $D_{S F R A}$ of a two-area power system are calculated. The system consists of two areas connected by a tie. Area $A$ consists of four thermal units and a wind farm. The overall capacity of Area $A$ and the wind farm is 3,000 and $600 \mathrm{MW}$ respectively. Area $B$ consists of four thermal units and the overall capacity is $2,000 \mathrm{MW}$. Condensation steam turbine generator is utilized in this case study. The transfer function of generator $i$ is $G_{i}(s)=1 /\left(T_{0} s+a\right) \cdot 1 /\left(T_{s} s+1\right)$, where $T_{s}$ is the time constant for hydraulic servomotor, $T_{0}$ is the time constant for high pressure cylinder volume. The values of these coefficients are $T_{s}=0.2 \mathrm{~s}, T_{s}=0.2 \mathrm{~s}, \delta_{i A}=\delta_{i B}=0.05, K_{A}=K_{B}=0.25$.

(1) Calculation of $D_{P F R A}: \alpha_{p f r}$ and $\delta_{i A}$ are the two main factors that influent the primary frequency regulation ability. By applying the variable control method, the impacts of each factor on $D_{P F R A}$ are analyzed. DPFRA under different values of $\delta_{i A}$ and $\alpha_{p f r}$ can be calculated using Matlab. The calculation results are shown in Table 3.

As shown in Table 3, the DPFRA of area $A$ increases as $\delta_{i A}$ decreases or as $\alpha_{p f r}$ increases. Concrete analysis results are as follows:
Table 3. $D_{P F R A}$ at different $\delta_{i A}$ and $\alpha_{p f r}$

\begin{tabular}{lllll}
\hline & & $\delta_{i A}=0.03$ & $\delta_{i A}=0.04$ & $\delta_{i A}=0.05$ \\
\hline$\alpha_{p f r}(\%)$ & 10 & 3.3155 & 2.4854 & 1.9881 \\
& 20 & 6.6310 & 4.9709 & 3.9761 \\
& 30 & 9.9466 & 7.4563 & 5.9642 \\
& 40 & 13.2621 & 9.9418 & 7.9523 \\
& 50 & 16.5776 & 12.4272 & 9.9403 \\
& 60 & 19.8931 & 14.9127 & 11.9284 \\
& 70 & 23.2086 & 17.3981 & 13.9164 \\
& 80 & 26.5242 & 19.8836 & 15.9045 \\
\hline
\end{tabular}

a) DPFRA is in direct proportion to $\alpha_{p f r}$ when $\delta_{i A}$ stays invariant. When the proportion of primary frequency regulation units $\alpha_{p f r}$ changes from $p$ to $q \%$. D PFRA changes from the original value $x$ to $(q / p) \cdot x$.

b) When $\alpha_{p f r}$ has an invariant value of $w$, the relationship between $D_{P F R A}$ and $\delta_{i A}$ can be described as:

$D_{P F R A}=\frac{w}{\delta_{i A}}$

(2) Calculation of $D_{S F R A}$ : Proportion of thermal units involved in secondary frequency regulation and $K_{A}$ are two main factors affect the secondary frequency regulation. The effects of each factor on $D_{S F R A}$ are studied using the variable control method. DSFRA under different values of $\alpha_{s f r}$ and $K_{A}$ are calculated which is shown in Table 4.

Table 4. $D_{S F R A}$ at different $K_{A}$ and $\alpha_{s f r}$

\begin{tabular}{lllll}
\hline & & $K_{A}=0.25$ & $K_{A}=0.5$ & $K_{A}=0.75$ \\
\hline$\alpha_{s f r}(\%)$ & 10 & 5.9818 & 15.0133 & 23.8114 \\
& 20 & 11.9636 & 30.0267 & 47.6227 \\
& 30 & 17.9455 & 45.0459 & 73.4341 \\
& 40 & 23.9273 & 60.0533 & 95.2454 \\
& 50 & 29.9091 & 75.0667 & 119.0568 \\
& 60 & 35.8909 & 90.0800 & 146.8642 \\
& 70 & 41.8727 & 105.0933 & 166.6795 \\
& 80 & 47.8545 & 120.1067 & 190.4909 \\
\hline
\end{tabular}

From Table 4, $D_{S F R A}$ of area $A$ increases as $\alpha_{s f r}$ increases. Concrete analysis results are as follows:

a) $D_{S F R A}$ is in direct proportion to $\alpha_{s f r}$ when $K_{A}$ stays invariant. When the proportion of primary frequency regulation units $\alpha_{s f r}$ changes from $p$ to $q \%, D_{S F R A}$ changes from the original value $x$ to $(q / p) \cdot x$.

b) When $\alpha_{s f r}$ is invariant, $D_{S F R A}$ increases as $K_{A}$ increases. When $K_{A}$ increases from 0.25 to $0.5, D_{S F R A}$ increases to 2.5 times of the previous value. When $K_{A}$ increases from 0.25 to $0.75, D_{S F R A}$ increases to nearly 4 times of the previous value.

Grid operators mainly concerned about the risk of exceeding the frequency deviation limits due to wind power fluctuations for the next dispatch cycle. DPFRA and DSFRA are two practical indexes to evaluate the grid's ability of smoothing out wind power fluctuations. 


\section{Applications of DPFRA and DSFRA on Power System Operation}

This section outlines how $D_{P F R A}$ and $D_{S F R A}$ are utilized in power system operation. Figure 7 illustrated the major steps of the method based on the test system of Figure 6 .

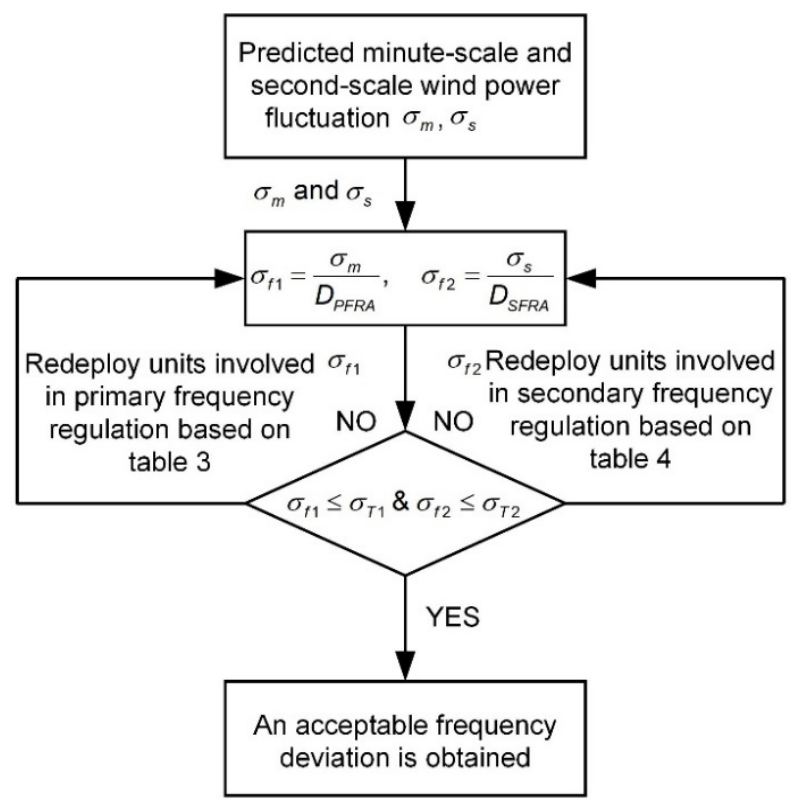

Figure 7. Method of utilizing DPFRA and $D_{S F R A}$ on power system operation.

As shown in Figure 7, after wind power fluctuations are predicted by Equations (2) and (3), DPFRA and DSFRA can be computed by Equations (20) and (21). System frequency deviation due to different time-scale wind power fluctuations can be obtained from Equations (9) and (11). If the frequency deviations exceed the threshold values, the operator can adjust the proportion of units participating in frequency regulation in the following dispatch cycle until an acceptable frequency deviation is obtained.

Four case studies based on the two-area system in Figure 6 are presented:

Initial operation condition of the system: the units participating in primary frequency regulation in area $A$ and $B$ account for 10 and $20 \%$ respectively, the units participating in secondary frequency regulation in area $A$ and $B$ account for 10 and $20 \%$ respectively. $\delta_{i A}=\delta_{i B}=0.05, K_{A}=K_{B}=0.25$. Acceptable grid frequency deviation is limited to $\pm 0.02 \mathrm{~Hz}$.

Based on the method in Figure 7, four cases study are presented to decrease frequency deviation. The operation conditions of each case are shown as follows:

Operation condition of Case I: $\alpha_{w}=20 \% ; \alpha_{p f r}$ increases from 10 to $20 \% ; \sum_{i=1}^{M 2} \alpha_{i A}$ increases from 10 to $20 \%$.

Operation condition of Case II: $\alpha_{w}=20 \% ; \sum_{i=1}^{M 1} \alpha_{i A}$ increases from 10 to $20 \% ; K_{A}$ increases from 0.25 to 0.5 .

Operation condition of Case III: $\alpha_{w}=20 \% ; \sum_{i=1}^{M 2} \alpha_{i A}$ increases from 10 to $20 \% ; \delta_{i A}$ decreases from 0.05 to 0.03 .

Operation condition of Case IV: $\alpha_{w}=20 \% ; K_{A}$ increases from 0.25 to $0.5 ; \delta_{i A}$ decreases from 0.05 to 0.03 .

Simulations are performed using MATLAB under different operating conditions. Corresponding frequency deviation curves are shown in Figure 8. Figure 8a shows the frequency deviation under the initial operation condition. Figure $8 \mathrm{~b} \sim$ $8 \mathrm{e}$ show the frequency deviations under operation conditions of each case respectively. As seen from Figure 8a, grid frequency deviation cannot be maintained within a stable range of $\pm 0.02 \mathrm{~Hz}$ when wind power accounts for $20 \%$ in grid.

(a) frequency deviation of initial condition

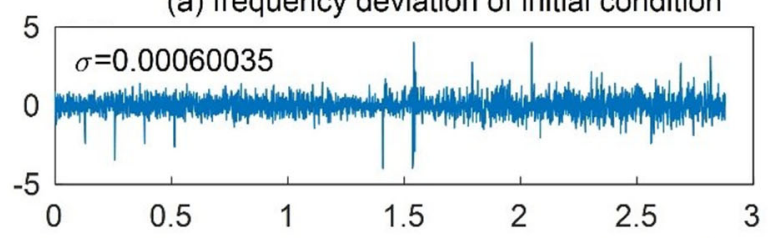

(b) frequency deviation of CASE I $\times 10^{4}$

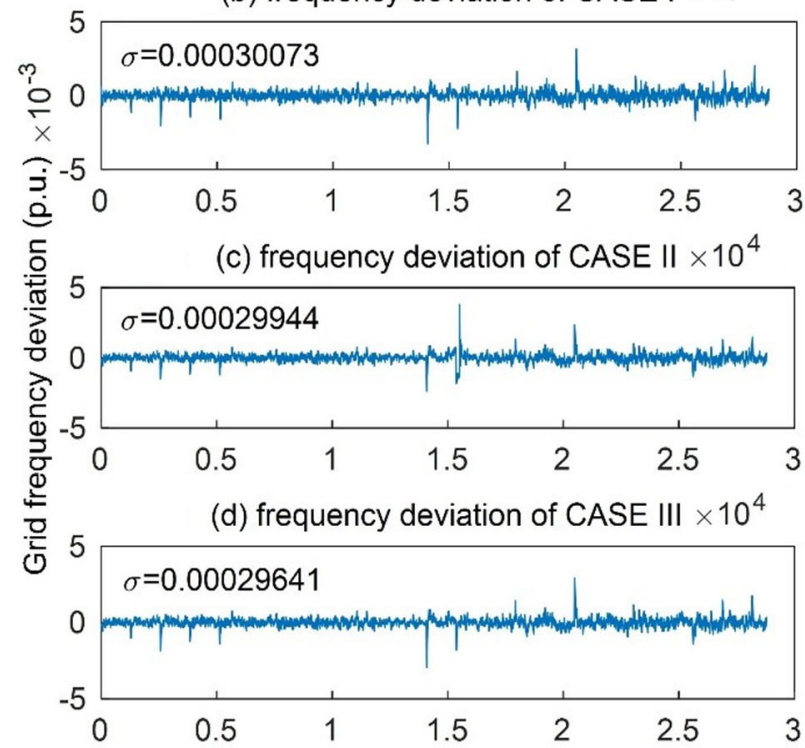

(e) frequency deviation of CASE IV $\times 10^{4}$

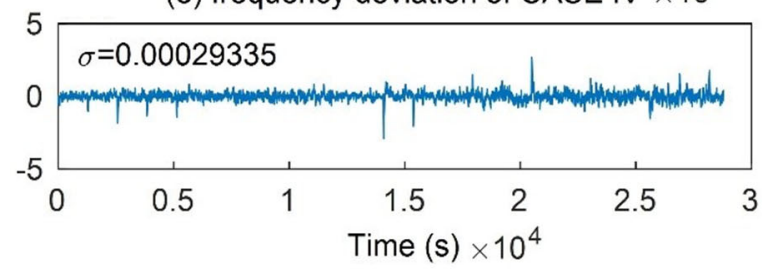

Figure 8. Frequency deviation of Area $A$ in different cases. (a) frequency deviation of initial condition; (b) frequency deviation of CASE I; (c) frequency deviation of CASE II; (d) frequency deviation of CASE III; (e) frequency deviation of CASE IV.

Under the operation condition of Case I, $D_{P F R A}$ increases from 1.9881 to 3.9761 and $D_{S F R A}$ increases from 5.9818 to 11.9636. Under operation condition of Case II, DPFRA increases from 1.9881 to 3.9761 and $D_{S F R A}$ increases from 5.9818 to 
15.0133. Under operation condition of Case III, DPFRA increases from 1.9881 to 3.3155 and $D_{S F R A}$ increases from 5.9818 to 11.9636. Under operation condition of Case IV, $D_{P F R A}$ increases from 1.9881 to 3.3155 and $D_{S F R A}$ increases from 5.9818 to 15.0133 .

$D_{P F R A}$ and $D_{S F R A}$ increase from the initial value in Case I Case IV. The standard deviations of system frequency deviation under each case are shown in Figure 8. The standard deviation of system frequency deviation reduces from 0.00060035 to $0.00030733,0.00029944,0.00029641$ and 0.00029335 , respectively.

\section{Conclusions}

This work presents a novel method for assessing the influence of stochastic wind power fluctuations on system frequency deviation. This research has the following advantages:

1) Wind power fluctuation model. Stochastic wind power fluctuations are fully analyzed in multi-time scales by the wavelet methods. A power-law model with three parameters is presented. The model is available for predicting both second and minute time-scale wind power fluctuations in time domain.

2) Quantitative frequency deviation assessment. The relationship between the stochastic wind power and grid frequency deviation is analyzed. Two practical indexes are defined to evaluate the grid's ability of smoothing out wind power fluctuations. The evaluation results help to verify whether the performance of power system satisfies grid codes.

3) Wide adaptability. Based on the online calculation results of $D_{P F R A}$ and $D_{S F R A}$, a strategy for deploying appropriate thermal units participating in frequency regulation is presented. Cases study show that this strategy is adaptable for decreasing grid frequency deviation.

In summary, this method is particularly valuable and practical for interconnected grids with a large amount of wind power integration.

Acknowledgments. This work was supported by the Key R\&D Project of China under grant number 2016YFB0901900, the National Natural Science Foundation of China (Grant No. 51676054).

\section{Nomenclature}

M1 Number of thermal units that participating in primary frequency regulation in area $A$.

M2 Number of thermal units that participating in secondary frequency regulation in area $A$.

$N 1 \quad$ Number of thermal units that participating in primary frequency regulation in area $B$.

$N 2 \quad$ Number of thermal units that participating in secondary frequency regulation in area $B$.

$\delta_{i A} \quad$ Speed droop of generator $i$ in area $A$.

$\alpha_{i A} \quad$ Ratio of installed capacity of generator $i$ to installed capacity of area $A$.

$R_{i} \quad$ Coefficient of power budget of the units that participating in secondary frequency regulation. $\alpha_{p f r} \quad$ Proportion of thermal units participating in primary frequency regulation, $\alpha_{p f r}=\sum_{i=1}^{M 1} \alpha_{i A}$.

$\alpha_{s f r} \quad$ Proportion of thermal units participating in secondary frequency regulation, $\alpha_{p f r}=\sum_{i=1}^{M 1} \alpha_{i A}$.

$G_{i}(s) \quad$ Transfer function of generator $i$.

$T_{a i} \quad$ Time constant of rotor $i$, in $\mathrm{s}$.

$T_{a \Sigma} \quad$ Inertia time constant of electric power system.

$T_{A B} \quad$ Synchronizing torque coefficient.

$\beta \quad$ Coefficient of load-frequency characteristic.

$K_{A} \quad$ Integrator gain in AGC control of area $A$.

$B_{A} \quad$ Frequency bias factor in area $A$.

$\chi_{N L}(s) \quad$ Variation of system load in the frequency domain.

\section{References}

Al-Awami A.T. and El-Sharkawi M.A. (2011). Coordinated trading of wind and thermal energy. IEEE Trans. Sustainable Energy, 2(11), 277-287. https://doi.org/10.1109/TSTE.2011.2 111467

Khosravi A., Nahavandi S., and Creighton D. (2013). Prediction intervals for short-term wind farm power generation forecasts. IEEE Trans. Sustainable Energy, 4(8), 602-610. https://doi.org/10. 1109/ TSTE.2012.2232944

Luo C. and Ooi B.T. (2006). Frequency deviation of thermal power plants due to wind farms. IEEE Trans. Energy Convers. 21(12), 708716. https://doi.org/10.1109/TEC.2006.8 74210

Luo C., Far H.G., Banakar H., and Ooi B.T. (2007). Estimation of wind penetration as limited by frequency deviation. IEEE Trans. Energy Convers., 3(6), 783-791. https://doi.org/10.1109/TEC.2006. 881082

Wu C.B., Huang G.H., Li W., Zhen J.L., and Ji L. (2016). An inexact fixed-mix fuzzy-stochastic programming model for heat supply management in wind power heating system under uncertainty. $J$. Cleaner Prod., 112, 1717-1728. https://doi.org/10.1016/j.jclepro. 2015.04.061

Bouffard F. and Galiana F.D. (2008). Stochastic security for operations planning with significant wind power generation. IEEE Trans. Power Syst., 23(5), 306-316. https://doi.org/10.1109/TPWRS.2008. 919318

Galiana F.D., Bouffard F., Arroyo J.M., and Restrepo J.F. (2005). Scheduling and pricing of coupled energy and primary, secondary and tertiary reserves. IEEE Proc., 93(2), 970-983. https://doi.org/ 10.1109/JPROC.2005.857492

Bouffard F. and Ortega-Vazquez M. (2011). The Value of Operational Flexibility in Power Systems with Significant Wind Power Generation. Power and Energy Society General Meeting, 2011 IEEE, San Diego, CA, USA, 2011. https://doi.org/10.1109/PES.2011. 6039031

Banakar H., Luo C., and Ooi B.T. (2008). Impacts of wind power minutetominute variations on power system operation. IEEE Trans. Power Syst., 23(4), 150-160. https://doi.org/10.1109/TPWRS.2007. 913298

Lin J., Sun Y., Srensen P., and Li G. (2012). Method for Assessing Grid Frequency Deviation Due to Wind Power Fluctuation Based on TimeFrequency Transformation. IEEE Trans. Sustainable Energy, 3(2), 783-791. https://doi.org/10.1109/TST E.2011.2162639

Kisi O., Shiri J., Akbari N., Pour M.S., Hashemi A., and Teimourzadeh K. (2013). Modeling of dissolved oxygen in river water using artificial intelligence techniques. J. Environ. Inf., 22(2), 92101. https://doi.org/10.3808/jei.201300248

Xia L., Junyong L., and Youbo L. (2013). Optimal Distribution Model for System Reserve Capacity with Wind Power Connection Regarding Consistency Performance of Reserve Dispatch. Autom Electric Power Syst., 40-46. https://doi.org/10.7500/AEPS201208 048 
Ji L., Huang G.H., Huang L.C., Xie Y.L., and Niu D.X. (2016). Inexact stochastic risk-aversion optimal day-ahead dispatch model for electricity system management with wind power under uncertainty. Energy, 109, 920-932. https://doi.org/10.1016/j.energy. 2016.05. 018

Nazir Md S. and Bouffard F. (2012). Intra-hour Wind Power Characteristics for Flexible Operations. Power and Energy Society General Meeting, 2012 IEEE, San Diego, CA, USA, 2012. https:// doi.org/10.1109/PESGM.2012.6345495

Ortega-Vazquez M.A. and Kirschen D.S. (2009). Estimating the spinning reserve requirements in systems with significant wind power generation penetration. IEEE Trans. Power Syst., 24(2), 114124. https://doi.org/10.1109/TPWRS.2008.2004745

Suo M.Q., Li Y.P., Huang G.H., Deng D.L. and Li Y.F. (2013). Electric Power System Planning under Uncertainty Using Inexact Inventory Nonlinear Programming Method. J. Environ. Inf., 22(1), 49-67. https://doi.org/10.3808/jei.201300245

Elgerd O.I. and Fosha C.E. (1970). Optimum megawatt-frequency control of multiarea electric energy systems. IEEE Trans. Power Apparatus Syst., 89, 556-563. https://doi.org/10.1109/TPAS.1970. 292602

Luickx P.J., Delarue E.D., and D'Haeseleer W.D. (2009). Effect of the generation mix on wind power introduction. IET. Renewable Power Generation, 3, 267-278. https://doi.org/10.10 49/ietrpg.2008. 0061

Sorensen P., Cutululis N.A., and Vigueras-Rodrigue A. (2007). Power fluctuations from large wind farms. IEEE Trans. Power Syst., 22, 958-965. https://doi.org/10.1109/TPWRS.2007. 901615
Hu Q., Huang G. H., Cai Y. P., and Sun W. (2014). Planning of Electric Power Generation Systems under Multiple Un-certainties and Constraint -Violation Levels. J. Environ. Inf., 23(1), 55-64. https:// doi.org/10.3808/jei.201400257

Rahmani, M.A. (2015). The use of statistical weather generator, hybrid data driven and system dynamics models for water resources management under climate change. J. Environ. Inf., 25(1), 23-35. https://doi.org/10.3808/jei.201400285

Boutsika T. and Santoso S. (2012). Quantifying short-term wind power variability using the conditional range metric. IEEE Trans. Sustainable Energy, 3, 369-378. https://doi.org/10.1109/TSTE.2012. 2186617

Can W., Xu Z., Pinson P., Dong Z.Y., and Kit Po.W. (2014). Probabilistic Forecasting of Wind Power Generation Using Extreme Learning Machine. IEEE Trans. Power Syst., vol. 29, 1033-1044. https://doi.org/10.1109/TPWRS.2013.2287871

Daren Y. and Guo Y.F. (2004). The online estimate of primary frequency control ability in electric power system. CSEE Proc., 24, 72-76.

Yao Y., Huang G.H., and Lin Q.G. (2012). Climate change impacts on Ontario wind power resource. Environ. Syst. Res., 1(1). https://doi. org/2.10.1186/2193-2697-1-2

Tan Z.F., Sog Y.H., Shen Y.S., Zhang C., and Wang S. (2013). An Optimization-Based Study to Analyze the Impacts of Clean Energy and Carbon Emission Mechanisms on Inter-Regional Energy Exchange. J. Environ. Inf., 22(2), 123-130. https://doi.org/10.3808/ jei.201300251 